\title{
D-2-Hydroxyglutarate producing neo-enzymatic activity inversely correlates with frequency of the type of isocitrate dehydrogenase 1 mutations found in glioma
}

\author{
Stefan Pusch', Leonille Schweizer², Ann-Christin Beck', Johanna-Marie Lehmler', Susanne Weissert², Jörg Balss', \\ Aubry K Miller ${ }^{3}$ and Andreas von Deimling ${ }^{1,2^{*}}$
}

\begin{abstract}
Background: IDH mutations frequently occur in diffuse gliomas and result in a neo-enzymatic activity that results in reduction of a-ketoglutarate to D-2-hydroxyglutarate. In gliomas, the frequency of IDH1 mutations in codon 132 increases in the order R132L-R132S-R132G-R132C-R132H with R132H constituting more than 90\% of all IDH1 mutations.

Results: We determined the levels of D-2-hydroxyglutarate in glioma tissues with IDH1 mutations. D-2-hydroxyglutarate levels increased in the order of R132H-R132C-R132S/R132G/R132L. We expressed and purified IDH1 wild type and mutant protein for biochemical characterization. Enzyme kinetics of mutant IDH protein correlated well with D-2-hydroxyglutarate production in cells with $\mathrm{R} 132 \mathrm{H}$ exhibiting the highest and R132L the lowest $\mathrm{K}_{\mathrm{M}}$ for a-ketoglutarate. Addition of D-2-hydroxyglutarate to the medium of cell lines revealed an inhibitory effect at higher concentrations. Migration of LN229 increased at lower D-2-hydroxyglutarate concentrations while higher concentrations showed no effect.

Conclusion: These findings may suggest natural selection against the rare IDH1R132 mutations in human glioma due to toxicity caused by high levels of D-2-hydroxyglutarate.
\end{abstract}

Keywords: IDH1, 2-HG, D-2-hydroxyglutarate, Enzymatic activity, $\mathrm{K}_{\mathrm{M}}$, Astrocytoma, Oligodendroglioma

\section{Background}

Mutations in isocitrate dehydrogenases 1 or 2 (IDH1, IDH2) occur approximately in $75 \%$ of diffuse astrocytomas and oligodendroglial tumors [1-3], in $20 \%$ of acute myeloid leukemia (AML) [4,5], 50\% of chondrosarcoma [6,7], 20\% of intrahepatic cholangiocarcinoma [8] and in $20 \%$ of angioimmunoblastic T-cell lymphoma [9]. Besides overall frequency, also the type of IDH mutations differs in these tumor entities. In astrocytoma and oligodendroglioma more than $90 \%$ of all IDH mutations are of the IDH1R132H type [2], with the second most frequent type (approximately $4 \%$ of mutations) being

\footnotetext{
* Correspondence: Andreas.vonDeimling@med.uni-heidelberg.de ${ }^{1}$ German Consortium of Translational Cancer Research (DKTK), Clinical Cooperation Unit Neuropathology, German Cancer Research Center (DKFZ), INF 280, Heidelberg D-69120, Germany

${ }^{2}$ Department of Neuropathology, Institute of Pathology, INF 224,

Ruprecht-Karls-University Heidelberg, Heidelberg D-69120, Germany

Full list of author information is available at the end of the article
}

IDH1R132C. The remaining mutations split into other very rare IDH1 and IDH2 mutations. Quite differently, IDH2R140Q mutations are most frequent in AML $[5,10]$. In chondrosarcoma and intrahepatic cholangiocarcinoma, the $\mathrm{R} 132 \mathrm{C}$ alteration in IDH1 is the most frequent mutation $[6,8]$, while in angioimmunoblastic T-cell lymphoma mutations in IDH2 are predominant [9]. Common to all IDH mutations is a neo-enzymatic activity of the mutated proteins that results in reduction of $\alpha$-ketoglutarate $(\alpha-K G)$ to D-2-hydroxyglutarate (2-HG) with consumption of NADPH. The neo-enzymatic activity of mutated IDH proteins is believed to constitute the major tumorigenic mechanism of this alteration due to the inhibitory effect of 2-HG on $\alpha$-KG dependent dioxygenases. Among these, the TET hydroxylases are affected resulting in a severely altered methylation pattern of DNA $[11,12]$ conferring an unknown advantage to these tumor cells. 
In astrocytoma and oligodendroglioma, we detected higher 2-HG levels in rare IDH1 mutations than in the most common IDH1R132H alteration [13]. A similar observation was made for IDH2, with cells carrying mutations affecting R172 exhibiting higher 2-HG concentrations than those with mutations in R140 [14]. These findings suggest different enzyme activity grades for the variant IDH mutants.

To explore the potential role of different mutation types in the formation of astrocytomas and oligodendroglial tumors, we determined the enzyme kinetics of wild type IDH1 and with the mutations R132H, R132C, R132G, R132S, R132L and R100Q followed by quantification of 2-HG in transfected cells and in human tumor tissues, and examined the effects on viability.

\section{Methods}

\section{FFPE samples, DNA extraction and IDH1 sequencing}

The collection of IDH1 mutant tumors consist out of previously published specimen $[1,2,15]$ and tumors collected at the neuropathology department of the pathological institute of Heidelberg.

The DNA extractions form FFPE samples were performed by hand using Invisorb Genomic DNA Kit II (Invitek, Berlin, Germany) or semi-automated using Maxwell ${ }^{\circledR}$ 16 FFPE Plus LEV DNA Purification Kit (Promega, Madison, USA) following the manufacturer's protocol.

The sequencing of published specimen was performed as described in the earlier publications. Our most recent probes were sequenced by generating a fragment of 212 bp length spanning the catalytic domain of IDH1 including codon 100 and 132 using 60 ng each of the sense primer IDH1-JM_f TGATGAGAAGAGGGTTGAGGA and the antisense primer IDH1-JM_r GCAAAATCACAT TATTGCCAAC. PCR using standard buffer conditions, 20 ng of DNA and GoTaq DNA Polymerase (Promega, Madison, USA) employed 35 cycles with denaturing at $95^{\circ} \mathrm{C}$ for $30 \mathrm{~s}$, annealing at $57^{\circ} \mathrm{C}$ for $40 \mathrm{~s}$ and extension at $72^{\circ} \mathrm{C}$ for $50 \mathrm{~s}$ in a total volume of $15 \mu \mathrm{l}$.

A total of $2 \mu \mathrm{l}$ of the PCR amplification product was submitted to the sequencing reaction using the BigDye Terminator v3.1 Sequencing Kit (Applied Biosystems, Foster City, USA). Twenty-five cycles were performed employing $12 \mathrm{ng}$ of the sense primer IDH1-JM_f TGAT GAGAAGAGGGTTGAGGA, with denaturing at $95^{\circ} \mathrm{C}$ for $30 \mathrm{~s}$, annealing at $57^{\circ} \mathrm{C}$ for $15 \mathrm{~s}$ and extension at $60^{\circ} \mathrm{C}$ for $240 \mathrm{~s}$. A second round of sequencing analysis was performed using the antisense primer IDH1-JM_r GCAAAATCACATTATTGCCAAC and the sequencing reaction conditions as described above. Sequences were determined using the semi-automated sequencer (ABI 3100 Genetic Analyzer, Applied Biosystems, Foster City) and the Sequence Pilot version 3.1 (JSI-Medisys, Kippenheim, Germany) software.

\section{IDH1 mutant generation and cloning}

IDH1 mutants were generated using the site directed mutagenesis method. Therefore, primers for each mutation were created and used on IDH1 wt cDNA in pDONR221 (DKFZ clone repository). Each mutation was confirmed by Sanger sequencing using the same procedure as described for the FFPE samples. The pDONR221 clones were used for all further LR-reactions into the described destination vectors. LR-reactions were performed following the manufacturers protocol (Invitrogen, Carlsbad, USA).

\section{IDH1 protein purification}

To purify the different IDH1 proteins, the cDNAs were transferred into pDEST15 (Invitrogen, Carlsbad, USA), an $E$. coli expression vector containing a N-terminal GST tag (Invitrogen, Carlsbad, USA). The pDEST15 vectors were then transfected into E.coli expression strain KRX (Promega, Madison, USA). The E.coli were then streaked out on lysogeny broth (LB)-plates containing Ampicillin (100 $\mu \mathrm{g} / \mathrm{ml}$, Sigma-Aldrich, St. Luis, USA) and incubated at $37^{\circ} \mathrm{C}$ overnight. Six clones from each construct were transferred into $6 \mathrm{ml}$ liquid LB containing $100 \mu \mathrm{g} / \mathrm{ml} \mathrm{Ampicillin} \mathrm{and} \mathrm{grown} \mathrm{over} \mathrm{night} \mathrm{at} 37^{\circ} \mathrm{C}$ in an orbital shaker (220 rpm). From these overnight cultures we used aliquots to prepare two small cultures $(10 \mathrm{ml})$ for induction experiments. We only induced one culture from each colony following the manufacturer's protocol (KRX protocol, Promega, Madison, USA). Briefly, inoculation of 1:100 of overnight culture, two hours incubation at $37^{\circ} \mathrm{C}$ and $220 \mathrm{rpm}$ in the orbital shaker followed by the induction with $0.1 \%$ L-Rhamnose ( $10 \%$ stock solution in water, AppliChem, Gatersleben, Germany) and four hours incubation at RT and $220 \mathrm{rpm}$. Subsequently both cultures of each clone were harvested by centrifugation and resuspendet in GST-Lysis buffer from the Pierce ${ }^{\odot}$ GST Spin Purification Kit (Thermo Scientific, Rockford, USA). Then they went through three freeze-thaw cycles with liquid nitrogen and a $15 \mathrm{~min}$ sonification step, followed by a $15 \mathrm{~min}$ centrifugation at $5000 \mathrm{rpm}$ on $4^{\circ} \mathrm{C}$. The supernatant was used for a SDS-PAGE (Invitrogen, Carlsbad, USA). A coomassie stain of the gel showed us which colonies yield best results. We then used these clones according to the protocol described above to prepare $200 \mathrm{ml}$ cultures. The supernatant of these cultures were then used for protein purification with the Pierce ${ }^{\odot}$ GST Spin Purification Kit (Thermo Scientific, Rockford, USA), following the manufacturer's protocol.

\section{IDH1 enzyme kinetics}

The measurement of the enzyme kinetics was performed with an Omega FluoStar (BMG Labtech, Ortenberg, Germany) equipped with a pump system, which was used to start the reaction, by adding $4 \mu \mathrm{g}$ protein to the reaction mixture. All measurements were performed in 
96-well plates (BD Falcon, Franklin Lakes, USA) in a total volume of $100 \mu \mathrm{l}$ at $37^{\circ} \mathrm{C}$. The reaction mixture consisted of Tris- $\mathrm{HCl} \mathrm{pH} 7.4$ (50 mM), $\mathrm{MgCl}_{2}(2 \mathrm{mM})$, $\mathrm{NaCl}$ (10 mM), BSA (0.05\%), NADP+/NAPDH (10 mM or concentration row), and isocitrate/ $\alpha-\mathrm{KG}$ (10 $\mathrm{mM}$ or concentration row). As a negative control we used the same mixture, but without isocitrate or $\alpha-K G$, depending on the $K_{M}$ to be obtained. After the start of the reaction, a one second double orbital shaking (500 rpm) was used to mix the reaction. Thereafter, every two minutes data was obtained from each well measuring the NADPH production or consumption (Ex. $340+/-10$ nm, Em. $440+/-10 \mathrm{~nm}$ ), depending on the reaction analyzed. All measurements were done in triplicate and at least 15 data points of each reaction were used for $K_{M}$ calculation. The mean of three independent measurements and the standard deviation of these is plotted for each $K_{M}$.

\section{D-2-hydroxyglutarate}

The 2-HG detection was performed with an enzymatic assay developed in our lab. Probe preparations and measurements were performed as described in [16].

D-2-hydroxyglutarate was obtained from Sigma Aldrich (St. Luis, USA) as disodium salt (catalogue number H8378).

Octyl-D-2-hydroxyglutarate was synthesized following the method reported by $\mathrm{Xu}$ et al. [12] with slight modifications (detailed protocol for D-2-hydroxyglutarate synthesis, see Additional file 1 and Additional file 2: Figure S1).

\section{Cell lines}

All cell lines were achieved from the ATCC and cultured under standard culture conditions $\left(37^{\circ} \mathrm{C}, 5 \% \mathrm{CO}_{2}\right)$ in DMEM medium with $1 \%$ Penicillin and Streptomycin and $10 \%$ fetal calf serum (all obtained from Gibco ${ }^{\circ}$ Invitrogen, Carlsbad, USA).

For the generation of IDH1R132H overexpressing cells, IDH1R132H in the destination vector pDEST26 (N-terminal 6x His Tag) was used. LN229 cells were transfected with IDH1R132H in pDEST26 vector by Fugene 6 (Promega, Madison, USA) followed by picking of single cell clones. Single cell clones were selected with $2 \mathrm{mg} / \mathrm{ml}$ Gentamycin (Invitrogen, Carlsbad, USA). Clones that survived selection were analyzed for their expression of IDH1R132H by western blot. Clones H3 and $\mathrm{H} 114$ were chosen for further analysis, due to their different and stable expression levels of IDH1R132H.

For all experiments with the inducible expression system we used normal cell culture medium, but exchanged normal FCS with 10\% Tet system approved FBS (Clontech, Mountain View, USA). To generate an inducible cell line we used the pT-REx-DEST system (Invitrogen, Carlsbad, USA). As a first step we transfected the cell line LN319 with pcDNA6/TR. From this transfection we generated single cell clones and tested their reliability by introducing EGFP in pT-REx-DEST30. We chose the clone with no GFP expression in tetracycline free media and with the highest expression after induction with $1 \mu \mathrm{M}$ Doxycycline (Sigma-Aldrich, St. Luis, USA). This clone 09 (K09) was then used for all further experiments.

For the experimental setup we used LN319 K09 transfected with IDH1 wild type (wt), the different IDH1 mutants, and GFP in pT-REx-DEST30. All cell lines were seeded as described under proliferation analysis. For each cell line two triplicates were seeded. One was induced with $1 \mu \mathrm{g} / \mathrm{ml}$ Doxycycline, the other one was treated with the comparable amount of solvent (DMSO, Sigma-Aldrich, St. Luis, USA).

To generate cell lines which express IDH1 wt and mutant proteins, we used cDNAs in pMXs-GW-IRES-BsdR and transfected them into HEK293T cells with FuGene ${ }^{\circ}$. Cells were subsequently put under selection pressure, by adding $4 \mu \mathrm{g} / \mathrm{ml}$ Blasticidine S (Sigma-Aldrich, St. Luis, USA). As control we used GFP in pMXs-GW-IRES-BsdR.

\section{Cell line analysis}

For cell number analysis CellTiterGlo (Promega, Madison, USA) was used in 96-well plate format. All cells were seeded at a density of 5,000 cells/well and subsequently treated. Measurements were performed at the indicated time points following the manufacturer's protocol.

All migration assays were performed with Ibidis Culture-Inserts. In each well of the insert, 50,000 cells were seeded. After $24 \mathrm{~h}$, the cells were treated with $10 \mu \mathrm{g} / \mathrm{ml}$ Mitomycin C (Sigma-Aldrich, St. Luis, USA) for $2 \mathrm{~h}$, to avoid the influence of proliferation in the assay. Afterward, the inserts were removed leaving a gap of $500 \mu \mathrm{m}+/-50 \mu \mathrm{m}$. Cells were washed with PBS once and thereafter normal medium was applied. This medium was subsequently substituted with $2-\mathrm{HG}$ at the given concentrations. The gap was microscopically documented at the start and the indicated time points. The pictures were analyzed with TScratch (http://chaton. ethz.ch/software/) and the area closed after the indicated time point was plotted in the graph.

The soft agar assays were performed in 6-well plates. The wells were prepared with a 1\% agar solution (Agar noble, US Biological, Salem, USA) as base agar. On top of this, 5,000 cells in $0.35 \%$ agar were seeded and grown for 14 days. The cells were stained with $0.00025 \%$ crystal violet solution and colonies $>1 \mathrm{~mm}^{2}$ were counted.

\section{Results}

\section{Distribution of IDH1 mutations in gliomas}

Including data from a previous study [2] and on ongoing routine analysis of all diffuse astrocytoma and oligodendroglioma in the Department of Neuropathology at the University Heidelberg, the present series of IDH1 


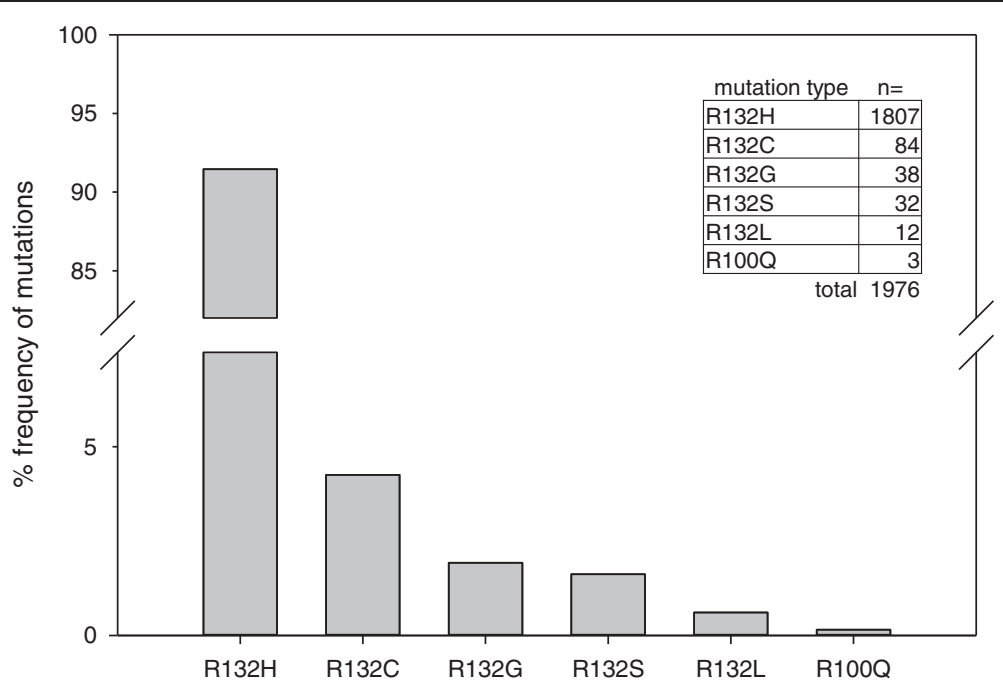

Figure 1 Distribution of IDH1 mutation types. Distribution of mutation types in the Heidelberg series of 1,976 diffuse astrocytomas and oligodendrogliomas with IDH1 mutations.

mutated gliomas comprises tumors from 1,976 patients. These 1,976 tumors represent only the immunohistochemical and sequence verified IDH1 mutant cases out of the whole collection of glial tumors in our department. The frequency of IDH1 mutations in these tumors decreases in the order $\mathrm{R} 132 \mathrm{H}$ (91.5\%), R132C (4.3\%), R132G (1.9\%), R132S (1.6\%), R132L (0.6\%) and R100Q (0.3\%). Incidences of the mutations are given in Figure 1.

\section{2-HG in tumor tissue}

We determined the amount of 2-HG in glioma tissue. The limiting step of our analyses was the low incidence of IDH1 mutations in gliomas other than R132H. Sufficient formalin fixed and paraffin embedded tissue was available from 9 tumors with R132C, 6 tumors with R132G and 1 each with R132S and R132L. Due to loss of 2-HG during the processing up to paraffin embedding [13], only approximate values for 2 -HG could be determined. Lowest 2-HG levels were detected in tumors with R132H followed by R132C and then R132G. The single tumors with R132S and R132L mutation also exhibited high levels of 2-HG. No 2-HG was detected in 5 control glioma samples without IDH1 mutation. The data is shown in Figure 2.

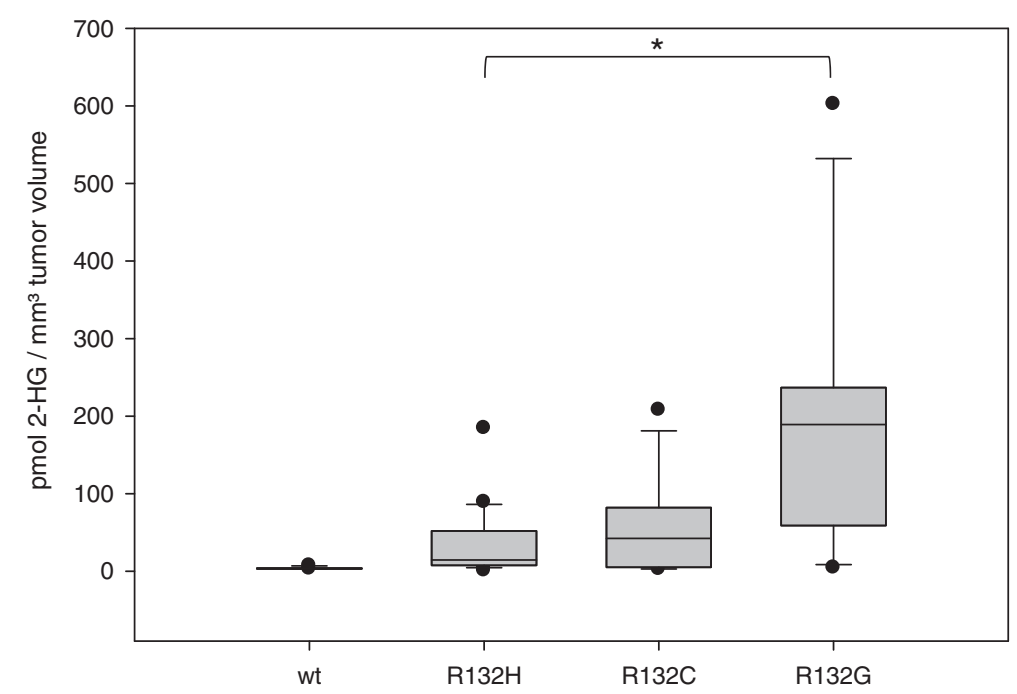

Figure 2 D-2-hydroxyglutarate levels in FFPE tissue. D-2-hydroxyglutarate levels in FFPE tissue from diffuse astrocytomas and oligodendrogliomas with IDH1 mutations. Shown are the levels of 6 IDH1 wt tumors, 25 with IDH1R132H, 8 with IDH1R132C and 7 with IDH1R132G. All mutant tumors show significant $(* *=p \leq 0.01$ ) higher $2-\mathrm{HG}$ levels than wildtype tumors, but only the difference between R132H and R132G mutation was statistically significant $\left({ }^{*}=p \leq 0.05\right)$. 


\section{Enzyme kinetics}

Recombinant expression of proteins with IDH1 mutations followed by purification enabled the determination of Michaelis constants for each mutant protein with the substrate $\alpha$-KG. A high $K_{M}$ of $\alpha-K G$ was observed for the $\mathrm{R} 132 \mathrm{H}$ mutation $(243.7 \mu \mathrm{M})$ whereas lower $\mathrm{K}_{\mathrm{M}}$ 's were seen for R132G $(67.4 \mu \mathrm{M})$, R132C (33.7 $\mu \mathrm{M})$, R132S $(26.1 \mu \mathrm{M})$ and R132L $(11.8 \mu \mathrm{M})$. Data is shown in Figure 3A.

The very rare $\mathrm{R} 100 \mathrm{Q}$ mutation exhibited a high $\mathrm{K}_{M}$ of $(3671.1 \mu \mathrm{M})$. This was the only mutated IDH1 protein for which a Michaelis constant for isocitrate was detected $\left(K_{M}=2724.0 \mu \mathrm{M}\right)$ (Figures 3A and 3B). To further validate this data, we transfected HEK293T cells with all different IDH1 variants and determined the cellular 2-HG level (Figure 3C). As expected the R132C mutant produced more 2-HG than $\mathrm{R} 132 \mathrm{H}$ mutant. Also, the other mutants R132G, R132S and R132L all produced more than $\mathrm{R} 132 \mathrm{H}$. However, the 2-HG concentration measured did not exactly correspond to the $\mathrm{K}_{\mathrm{M}}$ values determined. The 2-HG concentration from R100Q was as low as expected, ranging below that from $\mathrm{R} 132 \mathrm{H}$. To exclude the possibility that the different 2-HG concentrations are due to differential expression
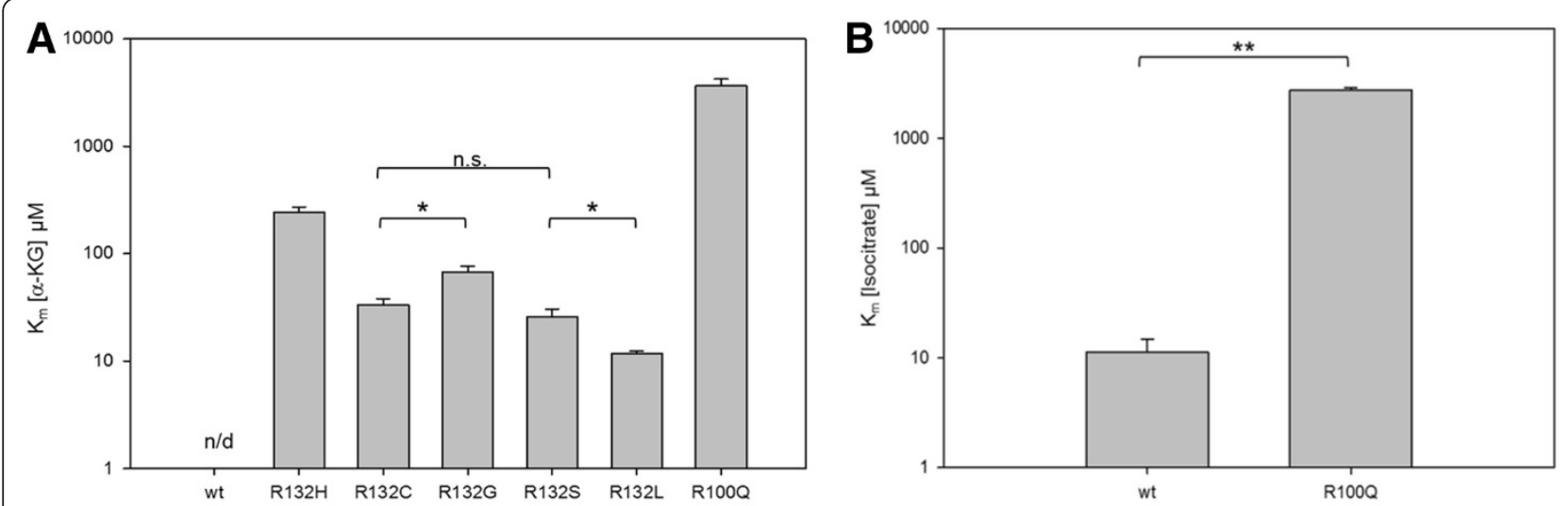

C

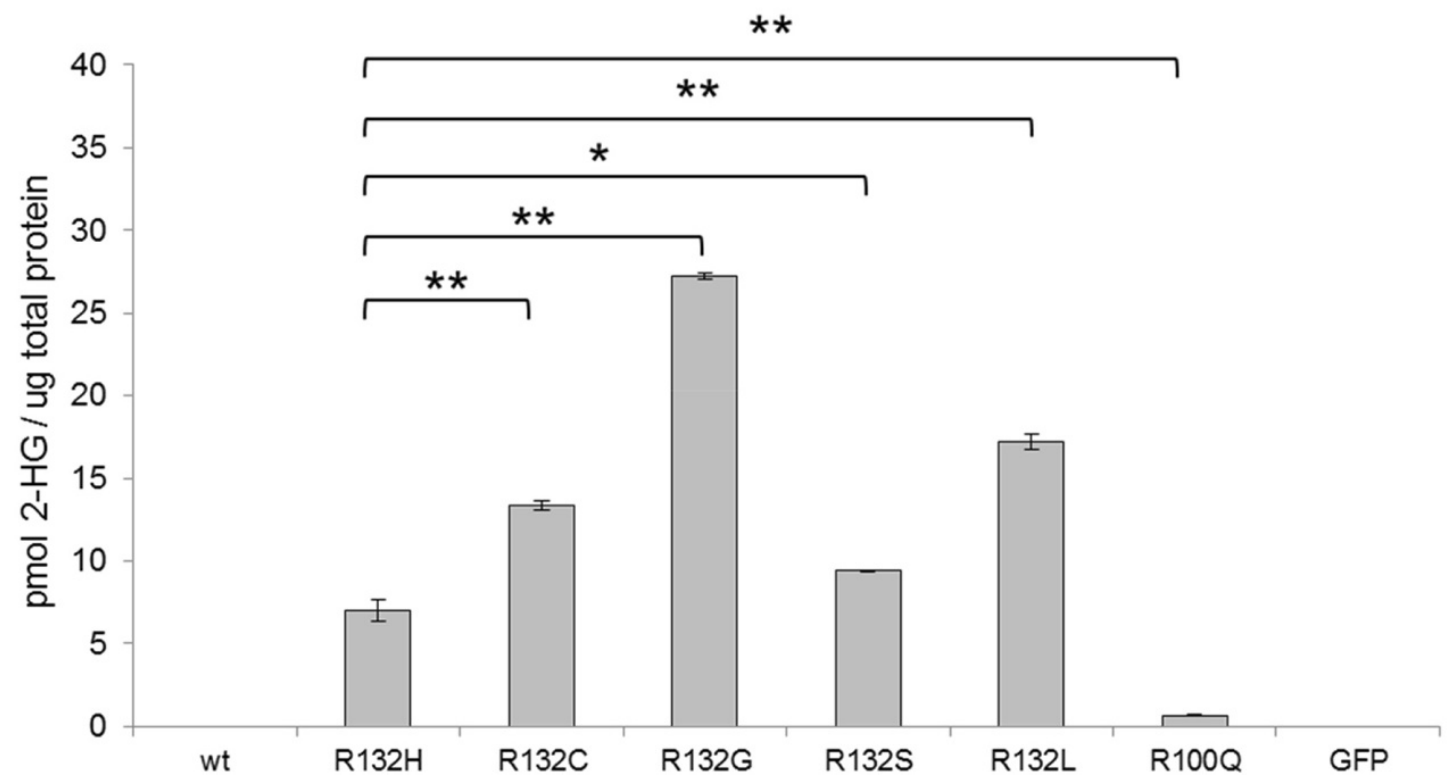

Figure $3 \mathrm{~K}_{M}$ values and D-2-hydroxyglutarate levels of IDH1 mutation types. $\mathrm{K}_{\mathrm{M}}$ values of IDH1 mutation types for a-ketoglutarate (A) and isocitrate (B). The $K_{M}$ value for a-ketoglutarate of $I D H 1$ wt and the $K_{M}$ values for isocitrate of all IDH1 ${ }^{R 132}$ mutations could not be determined. Therefore $K_{M}$ values of R132 mutations are not shown. All $K_{M}$ values are significantly different from each other with a $p$-value of $\leq 0.01$, if not otherwise stated ${ }^{*}=p \leq 0.05$ or n.s. $=$ not significant). All measurements were done in triplicate from different protein purifications. D-2-hydroxyglutarate levels in HEK293T cells ectopically expressing different IDH1 mutations and wild type IDH1 (C). Levels are measured in cells from one confluent dish of 6-well Plate $72 \mathrm{~h}$ after seeding. All D-2-hydroxyglutarate levels of the mutant IDH1 expressing cells are significantly higher ( $p \leq 0.01)$ than both controls ( $w$ t and GFP). The levels of all other R132 mutants are significantly higher than the one of R132H ( $p$-value: ${ }^{*}=p \leq 0.05$ and $*^{* *}=p \leq 0.01$ ). Only the level of R100Q is significant lower than the level of R132H ( $p$-value: ${ }^{* *}=p \leq 0.01$ ). 
of the respective mutant proteins or different protein stabilities, we performed a western blot demonstrating comparable protein quantity (Additional file 3: Figure S5).

\section{Cell viability dependence on 2-HG concentration}

Viability upon exogenous exposure to 2-HG was tested using immortalized human astrocytes, HEK293T cells, and seven glioma lines (LN18, LN229, LN319, A172, U87, U373, T98G) by addition of cell permeable octyl-2HG to medium [12]. To determine the concentration range to be used, we calculated the molar 2-HG concentrations in the tumor specimens from previous reports $[17,18]$ and our tumor material. For converting concentrations given in $\mu \mathrm{mol} / \mathrm{g}$ to molarities we assumed brain tumor tissue density to be similar to normal human brain tissue measuring $1.054+/-0.014 \mathrm{~g} / \mathrm{cm}^{3}$ [19]. On this basis, 2-HG concentrations in brain tumor tissues range from $0.1-126 \mathrm{mM}$. We, therefore, added cell permeable octyl-2-HG to cell medium in concentrations ranging from $100 \mu \mathrm{M}$ to $150 \mathrm{mM}$. All cell lines challenged perished at 2-HG concentrations of $7.5 \mathrm{mM}$ and above. Therefore, only data using octyl-2-HG concentrations ranging from $250 \mu \mathrm{M}$ to $7.5 \mathrm{mM}$ are shown in Figure 4A.

Due to the high toxicity of octyl-2-HG we switched to the less cell permeable sodium salt of 2-HG, because we aimed for more physiological concentrations in our experiments. We added this 2-HG to HEK293T, human astrocytes and LN229 in concentrations ranging from $500 \mu \mathrm{M}$ to $50 \mathrm{mM}$. Concentrations at the low end from $500 \mu \mathrm{M}$ to $1 \mathrm{mM}$ had no significant effect on proliferation. From $2.5 \mathrm{mM}$ to $50 \mathrm{mM}$, increasing toxicity was observed with LN229 being more resistant than HEK293T and human astrocytes (Figure 4B).

In order to test the effect of endogenous 2-HG we transfected LN229 with pDEST26 containing an IDH1R132H construct. Two clones $\mathrm{H} 3$ and $\mathrm{H} 114$ were established by single cell cloning, both stably producing $2-\mathrm{HG}$, albeit

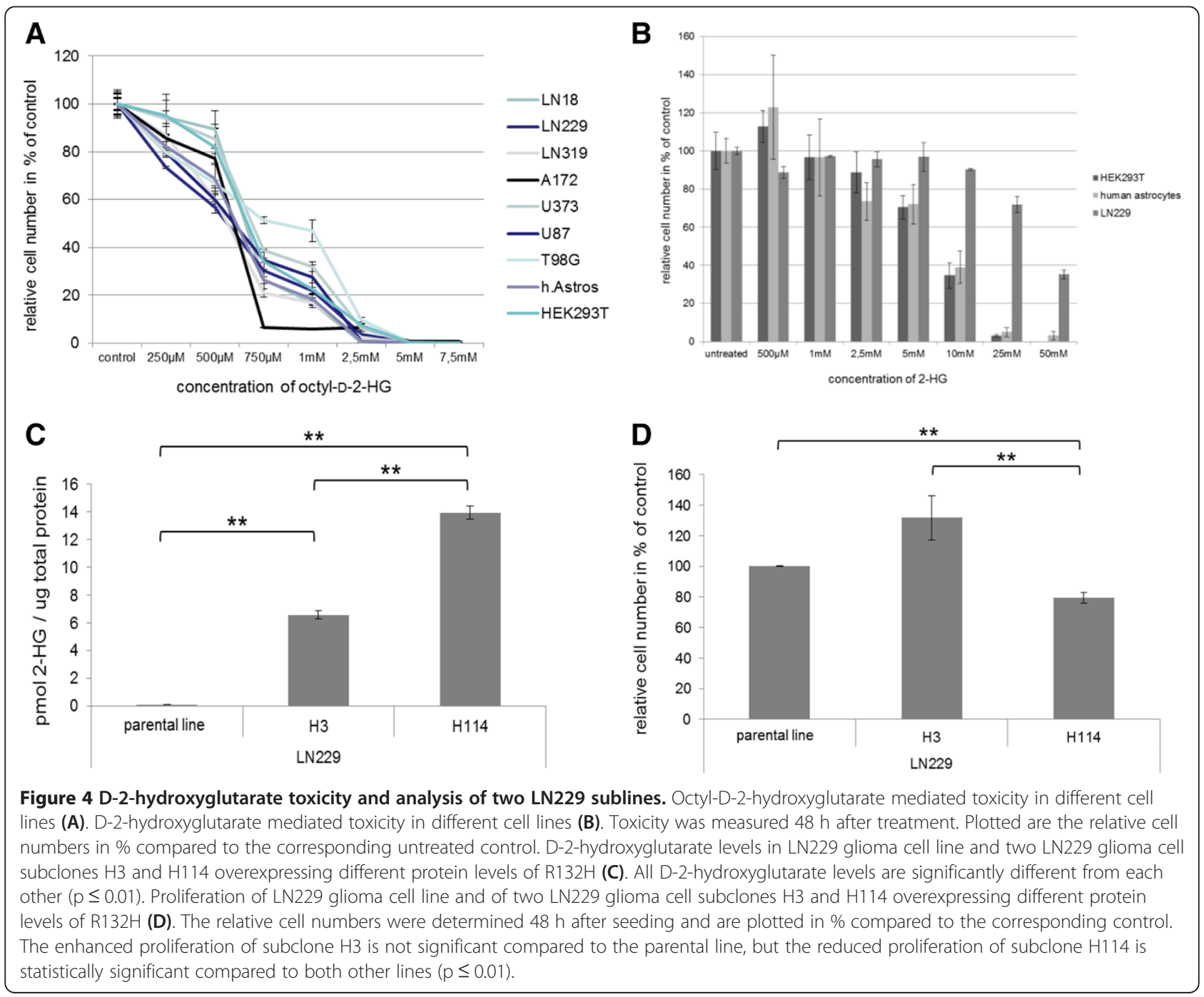


at different levels. Clone LN229 H3 contained 6.58 pmol $2-\mathrm{HG} / \mu \mathrm{g}$ total protein, a concentration not significantly affecting proliferation. In fact, a trend for higher proliferation was noted. In contrast, clone LN229 H114 containing $13.94 \mathrm{pmol} 2-\mathrm{HG} / \mu \mathrm{g}$ total protein exhibited a significant reduction of proliferation (Figures 4D).

\section{Concentration dependent toxicity of different IDH1 mutations}

We transfected in LN319 and HEK293T cells with pcDNA6/TR and selected single colonies. This was done to have cell lines with high expression of TR (tet repressor) sufficient for silencing pT-Rex-DEST30 prior to induction with Doxycycline.

We cloned IDH1 wt, all the R132 mutants and the R100Q mutant in pT-Rex-DEST30, and transfected these constructs into LN319 and HEK293T containing pcDNA6/ TR. Then, the expression of the respective mutant IDH protein was induced.

Induction of $\mathrm{R} 132 \mathrm{H}$ had no effect on proliferation in LN319, but in HEK293T it lead to a reduction in growth. Induction of all the mutations with lower $\mathrm{K}_{\mathrm{M}}$ for $\alpha-\mathrm{KG}$ resulted in reduced cell growth in both lines. As expected, expression of the R100Q mutation, producing low levels of 2-HG, also had no effect on growth in LN319. Neither expression of wt IDH1 protein nor of GFP affected cell growth in LN319. Results of growth kinetics for the different transfectants are shown in Figure 5.

\section{Effect of 2-HG concentration on migration and colony formation}

Wound healing assays were performed employing LN229 and different concentrations of 2-HG. In addition, we performed this assay employing LN229 H3 and LN229 H114 cells with ectopic expression of mutant R132H IDH protein at different levels. Addition of 2-HG at low concentration of $100 \mu \mathrm{M}$ resulted in enhanced migration, which was less pronounced at higher concentrations of $10 \mathrm{mM}$. LN229 H3 showed a migration rate higher than the parental LN229 while LN229 H114 did not differ from LN229 in this respect (Figure 6A).

Colony formation in soft agar was augmented upon exposure to 2-HG in moderate concentrations. Concentrations of $100 \mu \mathrm{M}$ and $10 \mathrm{mM} 2-\mathrm{HG}$ in medium both resulted in an increase of colonies by $61 \%$ and $59 \%$, respectively. LN229 H3 containing $6.58 \mathrm{pmol} 2-\mathrm{HG} / \mu \mathrm{g}$ total protein exhibited a 3 -fold increase in colony formation. In contrast, LN229 H114 containing 13.94 pmol 2-HG/ $\mathrm{gg}$ total protein did not form significantly more colonies than LN229 without treatment (Figure 6B).

\section{Discussion}

The IDH1 mutation of the R132H type by far outnumbers the other IDH1 mutations in diffuse astrocytic and oligodendroglial tumors. In order to provide a hypothesis for this lopsided distribution, we characterized biochemical features of different IDH1 mutations, analyzed native tumors and performed experiments addressing the effects of different mutations on in vitro systems.

Common to all IDH1 mutations is acquisition of a neoenzymatic activity with the ability to convert $\alpha-K G$ to 2-HG. Thus, we determined the Michaelis constants of mutated IDH proteins for the substrate $\alpha-K G$. We detected the highest $K_{M}$ for $\alpha-K G$ in protein carrying the $\mathrm{R} 132 \mathrm{H}$ mutation followed by R132G, R132C, R132S and R132L (Figure 3). While increased 2-HG levels are

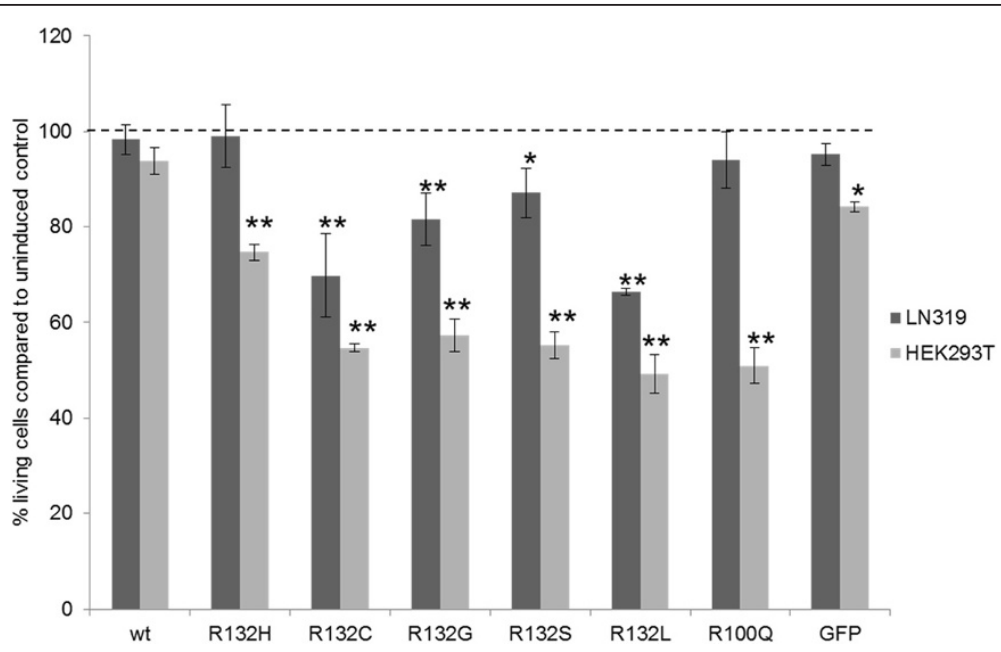

Figure 5 Proliferation analysis of LN319 glioma and HEK293T cells with and without induction of mutant IDH expression. Cell viability of LN319 glioma and HEK293T cells bearing tetracycline inducible constructs of different IDH mutations and controls $72 \mathrm{~h}$ after induction. Shown are the relative cell numbers compared to the corresponding uninduced controls. The significant differences are depicted in the figure with their corresponding to their $p$-values $\left(^{*}=p \leq 0.05\right.$ and $\left.{ }^{* *}=p \leq 0.01\right)$. 

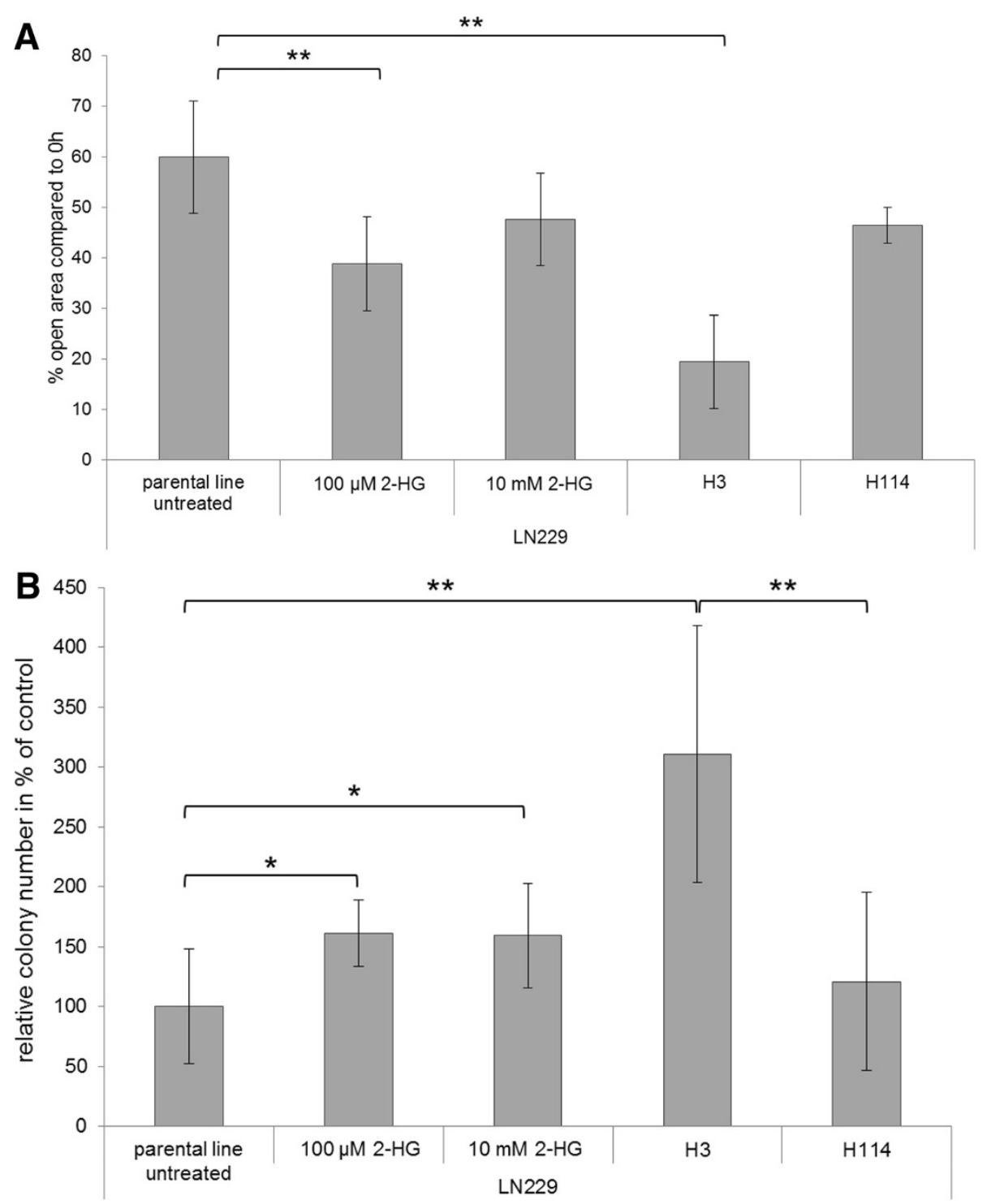

Figure 6 Migration and soft agar analysis of LN229 treated with D-2-hydroxyglutarate and LN229 subclones. Migration of LN229 and subclones H3 and H114 after 72 h. LN229 cells were treated with $100 \mu \mathrm{M}$ or 10 mM 2-HG. Subclones H3 and H114 expressed R132H protein in different amounts (A). Plotted is the open area after $24 \mathrm{~h}$ of incubation. Treatment with $100 \mu \mathrm{M} 2-\mathrm{HG}$ or ectopic expression of low amounts of $\mathrm{R} 132 \mathrm{H}$ protein (subclone $\mathrm{H} 3$ ) increased migration speed significantly ${ }^{* *}=\mathrm{p} \leq 0.01$ ). Colony formation in soft agar of the same cells in the same setting (B). Shown are the relative colony numbers compared to the untreated parental line (100\%). The 2-HG treatment could significantly $\left({ }^{*}=p \leq 0.05\right)$ increase colony formation independent of the used concentration. An even stronger increase $\left({ }^{* *}=p \leq 0.01\right)$ could be measured for subclone $\mathrm{H} 3$, but no difference could be detected for subclone $\mathrm{H} 114$.

expected to provide a selection advantage for early tumor cells, it should be kept in mind that high 2-HG levels may be toxic. Thus, positive discrimination for a mutation type resulting in a protein with moderate activity for features beneficial at low but potentially deleterious at high dose is well compatible with a selection process.

A beneficial effect of moderately increased 2-HG on proliferation (cell line LN229 H3, 6.58 pmol 2-HG/ $\mu$ g total protein) is demonstrated in Figure 4D. In contrast, strongly increased 2-HG levels (cell line LN229 H114,
13.94 pmol 2-HG/ $\mu \mathrm{g}$ total protein) did not favor proliferation. Similarly, migration could be increased for LN229 by $100 \mu \mathrm{M} 2-\mathrm{HG}$ in the medium and slightly less by $10 \mathrm{mM}$ 2-HG in the medium. Moreover, the cell line LN229 H3 with moderately induced 2-HG production exhibited increased migration compared to LN229. LN229 H114, with higher 2-HG production, did not migrate significantly faster than LN229 (Figure 6A). Likewise, colony formation was strongly supported by moderate 2-HG concentrations in the LN229 and subclone LN229 H3. In contrast, LN229 
H114, producing high levels of 2-HG, exhibited no benefit (Figure 6B).

To test the effect of 2-HG on viability of cells we added 2-HG, or the more cell permeable octyl-2-HG [12], to culture medium. In our models employing human astrocytes and HEK293T, $\mathrm{LD}_{50}$ for 2-HG was determined for concentrations between $5 \mathrm{mM}$ and $10 \mathrm{mM}$ while the $\mathrm{LD}_{50}$ for LN229 was approximately $50 \mathrm{mM}$ (Figure 4B). In the same cell lines and several more, the $\mathrm{LD}_{50}$ for octyl-2-HG was reached at approximately $750 \mu \mathrm{M}$ (Figure 4A). Moreover, transfection and induction of the different IDH1 mutations into LN319 cells demonstrated no influence on proliferation for $\mathrm{R} 132 \mathrm{H}$; however, the other mutations with lower $K_{M}$ for $\alpha-K G$ inhibited proliferation. These findings demonstrated considerable cell toxicity of 2-HG.

To further support this hypothesis we analyzed the amount of 2-HG in formalin fixed and paraffin embedded brain tumors harboring different IDH mutations. The limiting step of our analyses was the low incidence of IDH1 mutations other than $\mathrm{R} 132 \mathrm{H}$ in gliomas. The detection of moderately increased 2-HG levels in tumors with the IDH1R132H mutation and higher levels in the rare mutation types matched well with the different affinities for $\alpha-K G$ of the respective mutations.

Thus, in glioma cells we demonstrate beneficial effects of moderate 2-HG concentrations for proliferation, migration and colony formation and toxic effects on these readouts for high 2-HG concentrations. This would well match a model favoring an IDH1 mutation with an intermediate $K_{M}$ for $\alpha-K G$.

These findings may explain the strong preponderance of the IDH1R132H mutation type in glioma.

Both exo- and endogenous mechanisms affect types of mutation in DNA. Sporadic primary brain tumors in humans have not been shown to be associated with typical exogenous DNA damage such as ultraviolet-induced DNA-damage or that following exposure to carcinogens. It is unresolved whether accumulation of distinct mutations such as IDH1 originates from an endogenously mediated increase or a specific failure of repairing this distinct alteration. In low grade glioma, cytosine to thymidine transitions constitute the most frequent mutation type on the single nucleotide level [20]. R132H facilitated by a CGT to CAT change corresponds to a $\mathrm{C}$ to $\mathrm{T}$ transition on the reverse strand. On the other hand, $\mathrm{R} 132 \mathrm{C}$ facilitated by CGT to TGT is based on this transition on the coding strand. Neglecting potential repair of the $\mathrm{C}$ to $\mathrm{T}$ transition on the reverse strand during replication, $\mathrm{R} 132 \mathrm{H}$ and $\mathrm{R} 132 \mathrm{C}$ could be expected to occur with comparable frequency. This holds true for these mutations in acute myeloid leukemia [5,21]. In contrast, IDH1R132H dominates in diffuse gliomas and $\mathrm{R} 132 \mathrm{C}$ appears to be the most frequent mutation in chondrosarcoma [6,7] and intrahepatic cholangiocarcinoma $[8,22]$. Thus, the strong bias for
IDH1R132H mutation in astrocytoma and oligodendroglioma may support a selection bias for this alteration.

\section{Conclusion}

We determined significantly different enzymatic activities for distinct IDH1 mutations and provide a selection based hypothesis for the preponderance of the IDH1R132H mutation in astrocytoma and oligodendroglioma.

\section{Additional files}

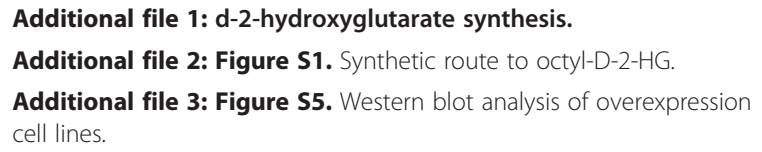

\section{Competing interests}

$A v D, J B$ and SP hold a patent for the enzymatic D-2-hydroxyglutarate detection assay. All other authors declare that they have no competing interests.

\section{Authors' contributions}

Conceived and designed the experiments SP. Generated mutant proteins and vectors SP, inducible cell line $J L S P$, over expression cell lines SW AB SP. Performed soft agar assay and migration assay LS, proliferation analysis $S P$, enzyme kinetics $A B$ JL JB, 2-HG detection assay $A B J B$, octyl-2-HG synthesis AM, database analysis AvD, statistical analysis LS SP. Wrote the manuscript AvD LS SP. All authors read and approved the final manuscript.

\section{Author details}

${ }^{1}$ German Consortium of Translational Cancer Research (DKTK), Clinical Cooperation Unit Neuropathology, German Cancer Research Center (DKFZ), INF 280, Heidelberg D-69120, Germany. Department of Neuropathology, Institute of Pathology, INF 224, Ruprecht-Karls-University Heidelberg, Heidelberg D-69120, Germany. ${ }^{3}$ Cancer Drug Development, German Cancer Research Center (DKFZ), INF 580, Heidelberg D-69120, Germany.

Received: 16 December 2013 Accepted: 24 January 2014

Published: 14 February 2014

\section{References}

1. Balss J, Meyer J, Mueller W, Korshunov A, Hartmann C, von Deimling A: Analysis of the IDH1 codon 132 mutation in brain tumors. Acta Neuropathol 2008, 116:597-602.

2. Hartmann C, Meyer J, Balss J, et al: Type and frequency of IDH1 and IDH2 mutations are related to astrocytic and oligodendroglial differentiation and age: a study of 1010 diffuse gliomas. Acta Neuropathol 2009, 118:469-474.

3. Yan H, Parsons DW, Jin G, et al: IDH1 and IDH2 mutations in gliomas. N Engl J Med 2009, 360:765-773.

4. Mardis ER, Ding L, Dooling DJ, et al: Recurring mutations found by sequencing an acute myeloid leukemia genome. N Engl J Med 2009, 361:1058-1066.

5. Paschka P, Schlenk RF, Gaidzik VI, et al: IDH1 and IDH2 mutations are frequent genetic alterations in acute myeloid leukemia and confer adverse prognosis in cytogenetically normal acute myeloid leukemia with NPM1 mutation without FLT3 internal tandem duplication. J Clin Oncol 2010, 28:3636-3643.

6. Amary MF, Bacsi K, Maggiani F, et al: IDH1 and IDH2 mutations are frequent events in central chondrosarcoma and central and periosteal chondromas but not in other mesenchymal tumours. J Pathol 2011, 224:334-343.

7. Arai M, Nobusawa S, Ikota H, Takemura S, Nakazato Y: Frequent IDH1/2 mutations in intracranial chondrosarcoma: a possible diagnostic clue for its differentiation from chordoma. Brain Tumor Pathol 2012, 29:201-206. 
8. Borger $\mathrm{D}$, Tanabe $\mathrm{K}$, Fan $\mathrm{K}$, et al: Frequent mutation of isocitrate dehydrogenase (IDH)1 and IDH2 in cholangiocarcinoma identified through broad-based tumor genotyping. Oncol 2012, 17:72-79.

9. Cairns RA, labal J, Lemonnier F, et al: IDH2 mutations are frequent in angioimmunoblastic T-cell lymphoma. Blood 2012, 119:1901-1903.

10. Ward PS, Patel J, Wise DR, et al: The common feature of leukemia-associated IDH1 and IDH2 mutations is a neomorphic enzyme activity converting alpha-ketoglutarate to 2-hydroxyglutarate. Cancer Cell 2010, 17:225-234.

11. Turcan S, Rohle D, Goenka A, et al: IDH1 mutation is sufficient to establish the glioma hypermethylator phenotype. Nature 2012, 483:479-483.

12. Xu W, Yang H, Liu Y, et al: Oncometabolite 2-hydroxyglutarate is a competitive inhibitor of alpha-ketoglutarate-dependent dioxygenases. Cancer Cell 2011, 19:17-30.

13. Sahm F, Capper D, Pusch S, Balss J, Koch A, Langhans C, Okun J, von Deimling A: Detection of 2-Hydroxyglutarate in formalin-fixed paraffin-embedded glioma specimens by gas-chromatography/mass-spectrometry. Brain Pathol 2012, 22:26-31.

14. Ward PS, Lu C, Cross JR, Abdel-Wahab O, Levine RL, Schwartz GK, Thompson $\mathrm{CB}$ : The potential for isocitrate dehydrogenase mutations to produce 2-hydroxyglutarate depends on allele specificity and subcellular compartmentalization. J Biol Chem 2013, 288:3804-3815.

15. Capper D, Weissert S, Balss J, et al: Characterization of R132H Mutation Specific IDH1 Antibody binding in brain tumors. Brain Pathol 2010, 20:245-254.

16. Balss J, Pusch S, Beck A-C, et al: Enzymatic assay for quantitative analysis of (D)-2-hydroxyglutarate. Acta Neuropathol 2012, 124:883-891.

17. Dang L, White DW, Gross S, et al: Cancer-associated IDH1 mutations produce 2-hydroxyglutarate. Nature 2009, 462:739-744.

18. Juratli TA, Peitzsch M, Geiger K, Schackert G, Eisenhofer G, Krex D: Accumulation of 2-hydroxyglutarate is not a biomarker for malignant progression in IDH-mutated low-grade gliomas. Neuro Oncol 2013, 15:682-690.

19. Barber TW, Brockway JA, Higgins LS: The density of tissues in and about the head. Acta neurologica Scandinavica 1970, 46:85-92.

20. Lawrence MS, Stojanov P, Polak P, et al: Mutational heterogeneity in cancer and the search for new cancer-associated genes. Nature 2013, 499:214-218.

21. Gross S, Cairns RA, Minden MD, et al: Cancer-associated metabolite 2-hydroxyglutarate accumulates in acute myelogenous leukemia with isocitrate dehydrogenase 1 and 2 mutations. J Exp Med 2010, 207:339-344.

22. Kipp BR, Voss JS, Kerr SE, et al: Isocitrate dehydrogenase 1 and 2 mutations in cholangiocarcinoma. Hum Pathol 2012, 43:1552-1558.

\section{doi:10.1186/2051-5960-2-19}

Cite this article as: Pusch et al.: D-2-Hydroxyglutarate producing neoenzymatic activity inversely correlates with frequency of the type of isocitrate dehydrogenase 1 mutations found in glioma. Acta

Neuropathologica Communications 2014 2:19

\section{Submit your next manuscript to BioMed Central and take full advantage of:}

- Convenient online submission

- Thorough peer review

- No space constraints or color figure charges

- Immediate publication on acceptance

- Inclusion in PubMed, CAS, Scopus and Google Scholar

- Research which is freely available for redistribution 